\title{
Natural Convection Inside a Rectangular Enclosure with Two Discrete Heat Sources Placed on Its Bottom Wall
}

\author{
Mehmet Saglam ${ }^{1}$, Bugra Sarper ${ }^{2}$, Orhan Aydin' \\ ${ }^{1}$ Karadeniz Technical University \\ Department of Mechanical Engineering \\ 61080 Trabzon, Turkey \\ ${ }^{2}$ Gumushane University \\ Department of Mechanical Engineering \\ 29100 Gumushane, Turkey \\ mehmetsaglam@ktu.edu.tr; oaydin@ktu.edu.tr; bugrasarper@gumushane.edu.tr
}

\begin{abstract}
In this study, natural convection of air inside a rectangular cavity with two discrete heat sources flush mounted on the bottom wall is investigated experimentally and numerically. The heaters are assumed to have different heat dissipation rates. In the experimental part, flow visualization and temperature measurements are performed. Numerical analysis is carried out for various values of modified Rayleigh number in the range of $2.3 \times 10^{4}$ and $2.3 \times 10^{6}$. The effect of the heat dissipation ratio of the heaters on the Nusselt number is investigated as well as streamlines, isotherms and heatlines. Experimental and numerical results are observed to be in a good agreement.
\end{abstract}

Keywords: Natural convection, electronics cooling, discrete heating, rectangular enclosure

\section{Introduction}

Natural convection in enclosures has attracted many researchers because of its wide range application areas such as cooling of electronic components, cooling and air-conditioning applications, energy efficient building design, solar energy systems and nuclear reactors.

There are numerous studies regarding natural convection in enclosures. Many of these works have studied differentially heated enclosures. Although it is important in the cooling of electronic equipments, studies on the localized heating in enclosures are limited. Aydin and Yang [1] investigated natural convection of air inside a square cavity heated from the bottom with a discrete heater and cooled from the sides for various Rayleigh numbers numerically. Corvaro and Paroncini [2] studied natural convection in a square enclosure heated from below and cooled from side walls numerically and experimentally with 2D-PIV and holographic interferometry. Banerjee et al. [3] studied natural convection inside a square cavity with two discrete heat sources flush mounted on the bottom wall and cooled from side walls numerically. Saravanan and Sivaraj [4] numerically examined natural convection in an air filled enclosure with a non-uniform discrete heat source placed on the center of the bottom wall and cooled from side walls. Zaman et al. [5] analysed buoyancy driven flow in an enclosure with two discrete heaters from the bottom numerically. Martyushev and Sheremet [6] numerically investigated transient natural convection and surface radiation in a closed cavity with conducting walls and a local heat source. Nardini et al. [7] studied the effects of protruding discrete heat source position placed on the bottom wall on natural convection heat transfer in a square cavity numerically and experimentally.

In this study, natural convection in a rectangular enclosure including two discrete heat sources on the bottom wall is investigated numerically and experimentally. The aim of this paper is to analyze the effects of heat dissipation ratio of the heaters on heat transfer and fluid flow. 


\section{Experimental Apparatus and Procedure}

The schematic of the problem under investigation and experimental set-up is shown in Fig. 1. The experimental set-up consists of a DC power supply, a data acquisition system, a hand type data logger, a diode laser, a CCD camera, an optical table, a fog generator, a thermostatic bath, a computer, an uninterruptable power supply and the test cell. An enclosure was manufactured with $31.75 \mathrm{~mm}$ height, $63.5 \mathrm{~mm}$ length and $152.4 \mathrm{~mm}$ depth. The aspect ratio of the enclosure is 0.5 . Enclosure walls are constructed with $10 \mathrm{~mm}$ thick plexiglass due to its transparency and low thermal conductivity. Heater surfaces are made of copper with $1.5 \mathrm{~mm}$ thickness and $12.7 \mathrm{~mm}$ width and $152.4 \mathrm{~mm}$ depth. $12.7 \mathrm{~mm}$ width of flexible heaters are used at the back of the copper surfaces. Ceiling of the enclosure is considered as the cold wall is made of 1.5 $\mathrm{mm}$ thick copper over which there is a water jacket. All of the copper surfaces are polished to minimize radiation heat transfer. The test cell is insulated with $60 \mathrm{~mm}$ foamboard to minimize heat losses to the environment.

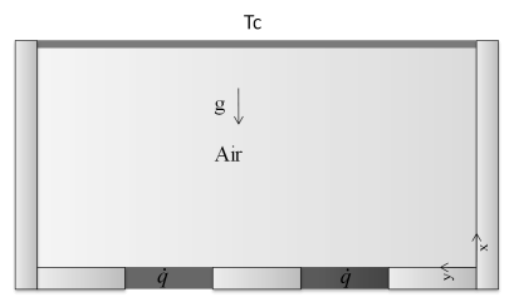

(a)

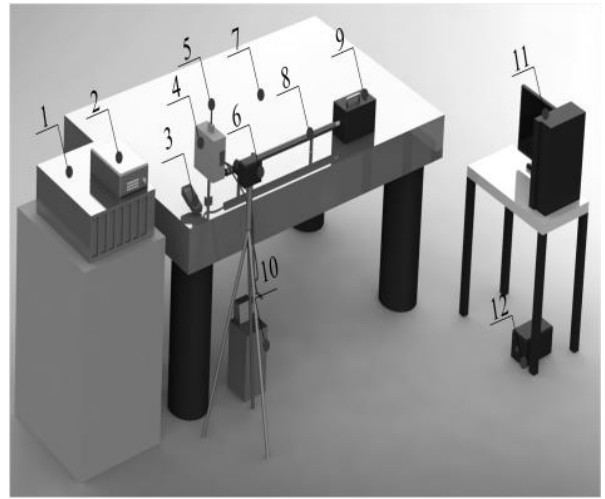

(b)
1. DC Power Source

2. Data Acquisition System

3. Hand Type Data Logger

4. Test Cell

5. Diode Laser

6. CCD Camera

7. Optical Table

8. Copper Pipe

9. Fog Genarator

10. Thermostatic Bath

11. Computer

12. UPS

Fig. 1: (a) Schematic of the problem, (b) Experimental set-up.

Temperature measurements are performed at the middle plane of the plexiglass walls and copper plates with $22 \mathrm{~T}$-type thermocouples. The test cell is placed on an optical table to minimize the vibration effects and oriented by using a digital protractor. The schematic of the thermocouple settlements and the picture of test cell are illustrated in Fig. 2.

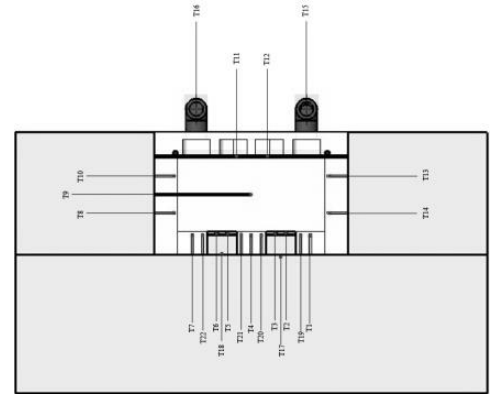

(a)

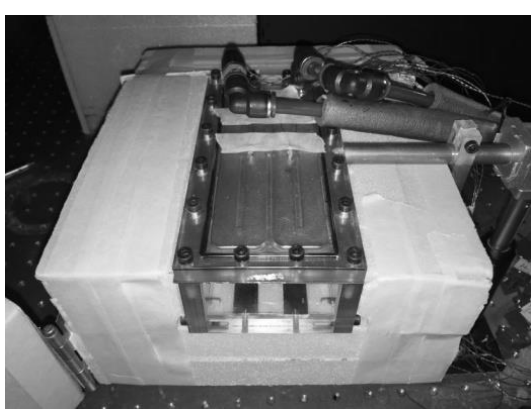

(b)

Fig. 2: Thermocouple settlements (a), photograph of the test section (b).

The power inputs of the heaters are calculated by using equation 1.

$$
P=I^{2} R=q_{m}^{\prime \prime} A_{s}
$$




$$
\begin{gathered}
I=\sqrt{\frac{q_{m}^{\prime \prime} A_{s}}{R}} \\
\dot{q}=\frac{P}{A_{s} t}
\end{gathered}
$$

Where, $I$ is the current supplied by DC power supply, $R$ is the resistance of the heater, $A_{s}$ is the surface area of the heaters, $t$ is the thickness of the heater. Also subscript $m$ denotes mean and $q_{m}^{\prime \prime}$ is the mean value of two surface heat flux values.

At the beginning of each experiment, temperature were kept at $24^{\circ} \mathrm{C}$ by an air-conditioner. Temperature of the cold wall is maintained at $24^{\circ} \mathrm{C}$ with a thermostatic bath. Temperature measurements are performed every five seconds during experiments. The flow is observed to reach steady state after 195 minutes. Smoke visualization technique is applied to display flow field. Safex F2010 fog generator and a $50 \mathrm{~mW}$ diode laser were used to visualize the flow domain. After temperatures have reached state, flow visualization process was started. The smoke was sent into the cavity by being passed into a long copper pipe to get the smoke cool down. It was waited for the smoke to get stable before taking the photos with a camera.

\section{Numerical Study}

In the numerical part of the study, continuity, momentum and energy equations are solved via ANSYS Fluent software. The flow is assumed to be two dimensional, steady and laminar. Fluid properties are considered as constant except density. Density change with temperature is modeled by adopting Boussinesq approximation. Although thermal conductivity of the plexiglass is low, experimental results show that conduction heat transfer cannot be neglected. Therefore, conduction heat transfer inside the plexiglass and insulation material is taken into account in the numerical analysis. Detailed comparisons were given between obtained experimental and numerical results. Thermophysical properties of the materials are seen in Table 1.

Table 1: Thermophysical properties of the materials.

\begin{tabular}{|c|c|c|c|c|}
\hline & $\boldsymbol{\rho}\left(\mathbf{k g} / \mathbf{m}^{3}\right)$ & $\mathbf{C}_{\mathbf{p}}(\mathbf{J} / \mathbf{k g K})$ & $\mathbf{k}(\mathbf{W} / \mathbf{m K})$ & $\boldsymbol{\varepsilon}$ \\
\hline Copper & 8978 & 381 & 387.6 & 0.05 \\
\hline Foamboard & 55 & 1500 & 0.035 & - \\
\hline Plexiglass & 1190 & 1470 & 0.19 & 0.85 \\
\hline
\end{tabular}

\subsection{Governing Equations}

The governing equations are non-dimensionalised with the dimensionless parameters as follows:

$$
(X, Y)=\frac{(x, y)}{H}, \quad(U, V)=\frac{(u, v) H}{\alpha}, \quad \theta=\frac{T-T_{C}}{q^{\prime \prime} H / k}, \quad P=\frac{p H^{2}}{\rho \alpha^{2}}, \quad R a=\frac{g \beta q_{m}^{\prime \prime} H^{4}}{k \vartheta \alpha}, \quad \operatorname{Pr}=\frac{\vartheta}{\alpha}
$$

The continuity, momentum and energy equations for a 2D, steady, incompressible and laminar flow are given as follows:

$$
\begin{gathered}
\frac{\partial U}{\partial X}+\frac{\partial V}{\partial Y}=0 \\
U \frac{\partial U}{\partial X}+V \frac{\partial U}{\partial Y}=-\frac{\partial P}{\partial X}+\operatorname{Pr}\left(\frac{\partial^{2} U}{\partial X^{2}}+\frac{\partial^{2} U}{\partial Y^{2}}\right)
\end{gathered}
$$




$$
\begin{gathered}
U \frac{\partial V}{\partial X}+V \frac{\partial V}{\partial Y}=-\frac{\partial P}{\partial Y}+\operatorname{Pr}\left(\frac{\partial^{2} V}{\partial X^{2}}+\frac{\partial^{2} V}{\partial Y^{2}}\right)+R a P r \theta \\
U \frac{\partial \theta}{\partial X}+V \frac{\partial \theta}{\partial Y}=\left(\frac{\partial^{2} \theta}{\partial X^{2}}+\frac{\partial^{2} \theta}{\partial Y^{2}}\right)
\end{gathered}
$$

Thus,

$$
N u=\frac{q_{\text {conv }}^{\prime \prime} H}{k\left(T-T_{c}\right)}
$$

\subsection{Boundary Conditions}

No-slip boundary condition is applied at all wall-to-fluid interfaces. Coupled boundary condition is implemented at all wall-to-fluid and wall-to-wall interfaces. At the outer surfaces of the insulation material, convection boundary condition is used. Convective heat transfer coefficient and temperature for the surrounding air is taken as $\mathrm{h}=7 \mathrm{~W} / \mathrm{m}^{2} \mathrm{~K}$ and $\mathrm{T}=24^{\circ} \mathrm{C}$. For the radiation calculation, emissivity values of the copper and plexiglass surfaces are 0.05 and 0.85 , respectively.

\subsection{Numerical Method}

Numerical study is performed using ANSYS Fluent software. The pressure based solver as suggested for low speed incompressible flows is preferred. For pressure-velocity coupling, SIMPLE algorithm is used. Discretization of momentum and energy equations is performed by second order upwind scheme and pressure interpolation is provided by PRESTO scheme. Radiation heat transfer between surfaces is taken into account by surface to surface radiation method (S2S) which assumes the surfaces gray and diffuse [8]. Convergence criterion is set to 10-6 for governing equations.

\subsection{Mesh Structure}

Non-uniform mesh structure is used in the numerical computations. It is seen from Fig. 3, smaller mesh size is used near the boundaries where temperature and density changes are substantial. In order to calculate temperature gradients in thermal boundary layer accurately, finer mesh is used in x direction while coarse mesh covers in y direction.

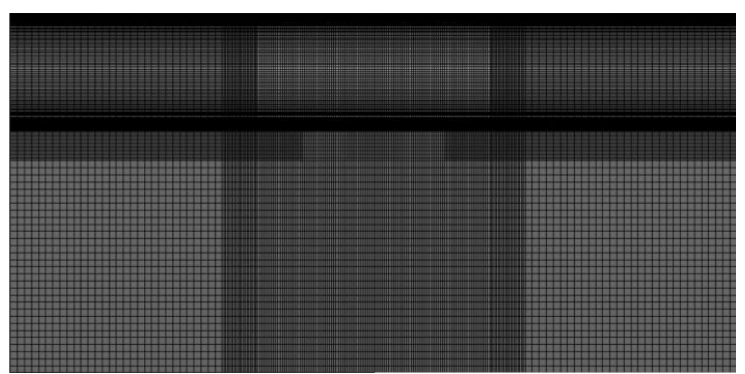

Fig. 3: Mesh structure of the numerical model.

A mesh independence study has been performed in order to make the numerical computations independent from the mesh structure. Three different mesh sizes have been used for the mesh independence study at $\mathrm{Ra}=7.7 \mathrm{x} 105$ for equal heat dissipation rate. Results are obtained for the average Nusselt number values on heater surfaces and the test shows that $101 \times 101$ mesh structure is seen enough for the computations (See Table 2).

Table 2: Mesh independence study results.

\begin{tabular}{|c|c|c|c|c|}
\hline & $\mathbf{N u}_{\mathbf{m}}$ (Left Heater) & $\mathbf{N u}_{\mathbf{m}}$ (Right Heater) & \multicolumn{2}{|c|}{ Change $(\%)$} \\
\hline $\mathbf{5 1 \times 5 1}$ & 4.685 & 4.685 & - & - \\
\hline $\mathbf{7 6 x 7 6}$ & 4.653 & 4.653 & 0.689 & 0.689 \\
\hline $\mathbf{1 0 1 \times 1 0 1}$ & 4.639 & 4.639 & 0.286 & 0.286 \\
\hline
\end{tabular}




\subsection{Heatlines Approach}

Heatlines approach was first presented by Kimura and Bejan to show energy transfer in flow field. As stated by the authors, "In convection problems, the energy transfer in flow field is sum of the heat diffusion and entalphy. For such a flow field, an $\boldsymbol{H}(\mathrm{x}, \mathrm{y})$ function could be defined where net energy transfer is zero through every constant $\boldsymbol{H}$ line" [9].

Mathematically, heatlines could be expressed as below:

$$
\frac{\partial H}{\partial y}=\rho c_{p} u\left(T-T_{0}\right)-k \frac{\partial T}{\partial x} ; \quad-\frac{\partial H}{\partial x}=\rho c_{p} v\left(T-T_{0}\right)-k \frac{\partial T}{\partial y}
$$

Where $\partial \boldsymbol{H} / \partial x$ and $\partial \boldsymbol{H} / \partial y$ denote the net energy flow except the surface radiation in $\mathrm{x}$ and $\mathrm{y}$ direction, respectively.

Subtracting these two equation side by side after taking first derivative, equation below could be obtained.

$$
\frac{\partial^{2} \boldsymbol{H}}{\partial x^{2}}+\frac{\partial^{2} \boldsymbol{H}}{\partial y^{2}}=\rho c_{p}\left[\frac{\partial\left(u\left(T-T_{0}\right)\right)}{\partial y}-\frac{\partial\left(v\left(T-T_{0}\right)\right)}{\partial x}\right]
$$

where $\mathrm{T}_{0}$ is the lowest temperature value in the flow field.

Boundary conditions for solution of this equation as followed; assuming no slip boundary condition on walls:

$$
\boldsymbol{H}=\oint_{C}-k \frac{d T}{d n} d s
$$

$\boldsymbol{H}(0, \mathrm{H})$ is taken as zero for the reference point. The heat equation and its boundary conditions are not nondimensionalized and solved by this way. After the heat function solved, it is non-dimensionalized with defined parameters as below.

$$
\boldsymbol{H}^{*}=\frac{\boldsymbol{H}}{\mathrm{q}_{\mathrm{m}}^{\prime \prime} \mathrm{H}}
$$

To solve the heatlines equation, first continuity, momentum and energy equations must be solved. Required datas for the solution of heat equation are exported from ANSYS Fluent after the problem being solved. A Matlab code is generated to solve the heat equation. Successive substitution method is applied until relative error value is lower than $10^{-6}$. Both correction of ANSYS Fluent solver and Matlab solver, solution datas compared with the study of Deng and Tang [10]. Streamlines, isotherms and heatlines obtained in the present study are presented in Fig. 4.

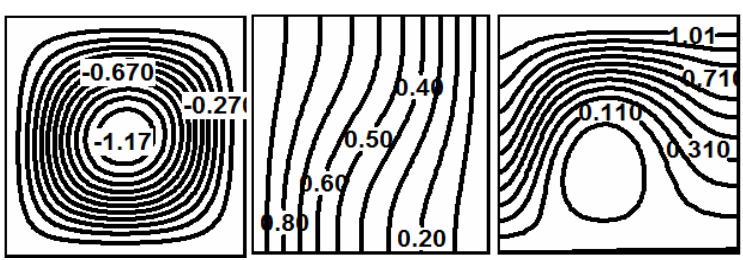

(a)
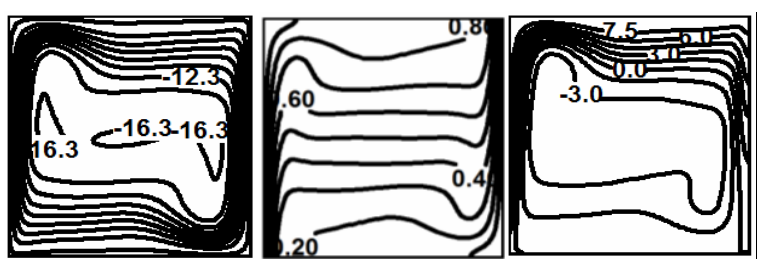

(b)

Fig. 4: Streamlines, isotherms and heatlines: (a) $\mathrm{Ra}=10^{3}$ and (b) $\mathrm{Ra}=10^{6}$. 


\section{Results and discussion}

Natural convection inside a rectangular cavity with two discrete heat sources placed on its bottom wall is investigated experimentally and numerically. Aspect ratio of the enclosure is 0.5 and the working fluid is air. Experiments are performed at $\mathrm{Ra} 7.7 \times 10^{5}$ while numerical part covers the Rayleigh numbers between $2.3 \times 10^{4}$ and $2.3 \times 10^{6}$ and heat dissipation ratios are $0.33,0.5$ and 1 while total heat generation rate is fixed.

Numerical and experimental results are compared with each other in terms of surface temperatures at the bottom wall and streamlines at $\mathrm{Ra}=7.7 \times 10^{5}$ for various heat dissipation ratios. It is seen from Fig. 5, dimensionless surface temperatures at the bottom wall are in a good agreement with each other and they follow the same trend. Additionally, two counterrotating cells take place in the enclosure in all situations but size of the cells vary with the heat dissipation ratio when experimental and numerical streamlines are examined (see Fig. 6). Numerical analysis is also performed for different Rayleigh numbers and presented with the figures below.

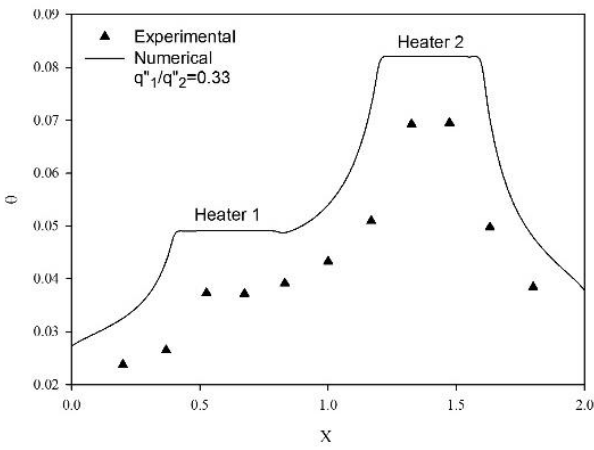

(a)

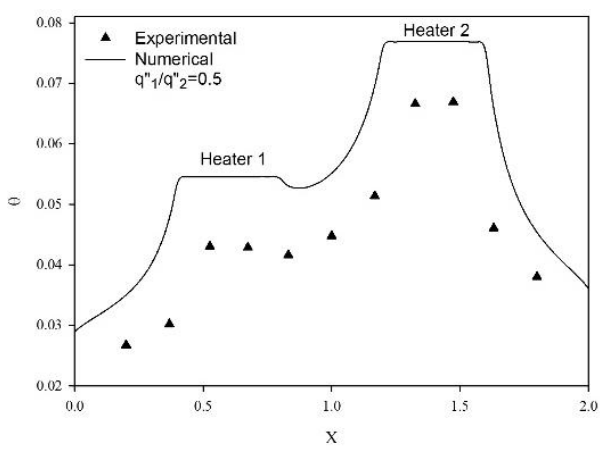

(b)

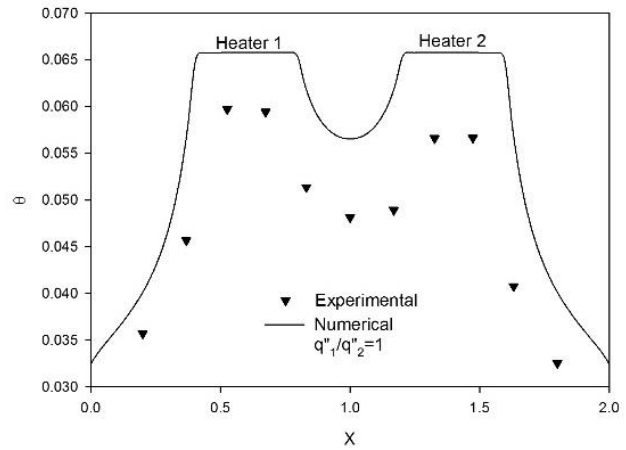

(c)

Fig. 5: Comparison of numerical and experimental temperature values on the bottom wall at $\operatorname{Ra} 7.7 \times 10^{5}$ : (a) $\mathrm{q}_{1}{ }_{1} / \mathrm{q}_{2}=0.33$, (b) $\mathrm{q}_{1}{ }_{1} / \mathrm{q}_{2}=0.5$, (c) $\mathrm{q}_{1}{ }_{1} / \mathrm{q}_{2}=1$ (Note the orientation of the coordinate system, See Fig. 1 ).

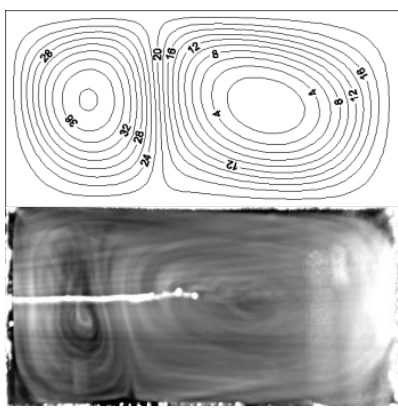

(a)

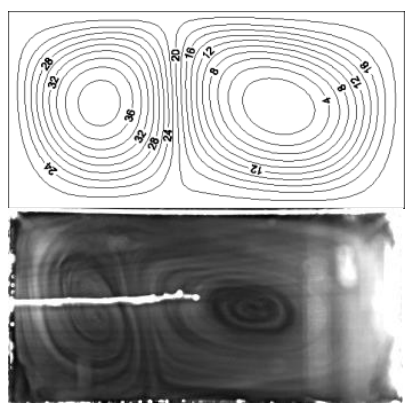

(b)

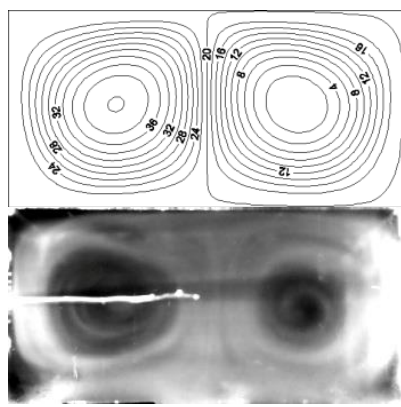

(c)

Fig. 6: Numerical and experimental streamlines at $R a=7.7 \times 10^{5}:$ (a) $\mathrm{q}_{1} / \mathrm{q}_{2}{ }_{2}=0.33$, (b) $\mathrm{q}_{1}{ }_{1} / \mathrm{q}_{2}=0.5$, (c) $\mathrm{q}_{1} / \mathrm{q}_{2}=1$.

Fig. 7 demonstrates the numerical streamlines, isotherms and heatlines at $\mathrm{Ra}=2.3 \times 10^{6}$. It is seen from the figure that, two counter-rotating cells occurs in the flow domain. When the heat dissipation ratio is 1 , cells in the main flow are symmetric to each other. A change in the heat dissipation ratio causes the cell size to change. Smaller cell takes place at the side of the stronger heat source. This is because of the position of the plume near the heat source whose dissipation rate is more. Heat flow corridor also shifts towards to stronger heat source's side, because higher heat will be transferred from stronger heat source. 


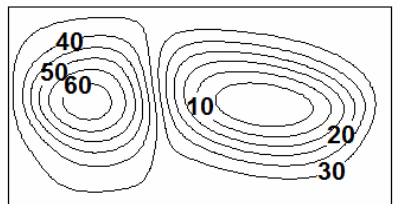

$(\mathrm{a}-1)$

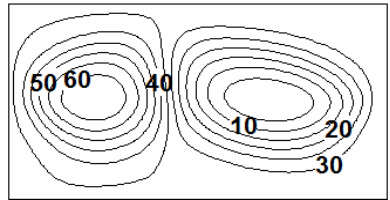

(b-1)

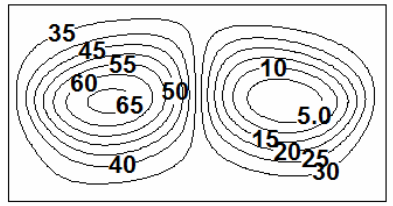

(c-1)

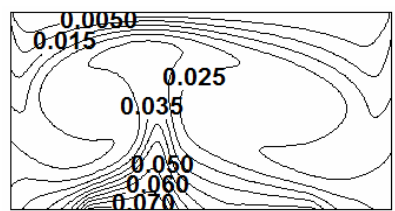

(a-2)

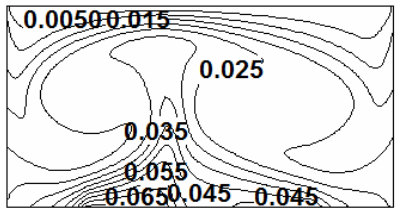

(b-2)

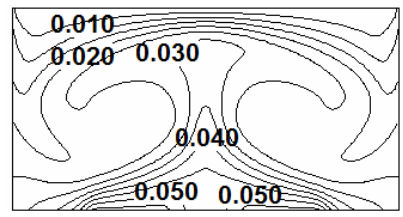

(c-2)

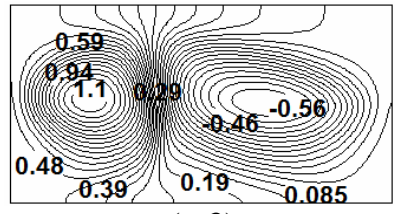

(a-3)

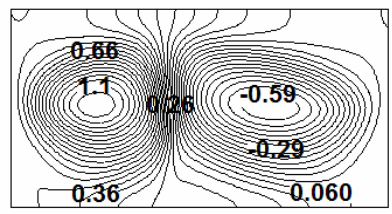

(b-3)

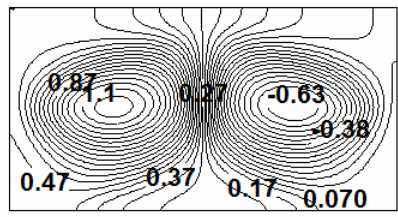

(c-3)

Fig. 7: (1) Streamlines, (2) isotherms and (3) heatlines at $R a=2.3 \times 10^{6}:$ (a) $\mathrm{q}_{1} / \mathrm{q}_{2}=0.33$, (b) $\mathrm{q}_{1}{ }_{1} / \mathrm{q}_{2}=0.5$, (c) $\mathrm{q}_{1} / \mathrm{q}_{2}=1$.

Fig. 8 shows the mean Nusselt number variation with heat dissipation ratio on the heater surfaces. It is seen from the figure, mean Nusselt number increases with Rayleigh number. When the heat dissipation ratio is 1, mean Nusselt number values at the right and left heater surfaces are equal to each other. A change in the heat dissipation ratio causes a decrease in mean Nusselt number values on both of the heater surfaces. Interestingly, Nusselt number value on the stronger heater is less than weaker one for high Rayleigh numbers. This may be explained with conduction heat transfer inside the enclosure walls. As seen from streamlines (Fig. 7), warm air on the bottom wall ascends because of density change and it starts to descend along the vertical walls after reached the cold surface. The descending cold air is in a contact with plexiglass enclosure walls and gets warmer before it touches the heater surfaces. Thus, convection heat transfer from the strong heater to the air decreases and lower Nusselt number values are obtained on the strong heater surface.

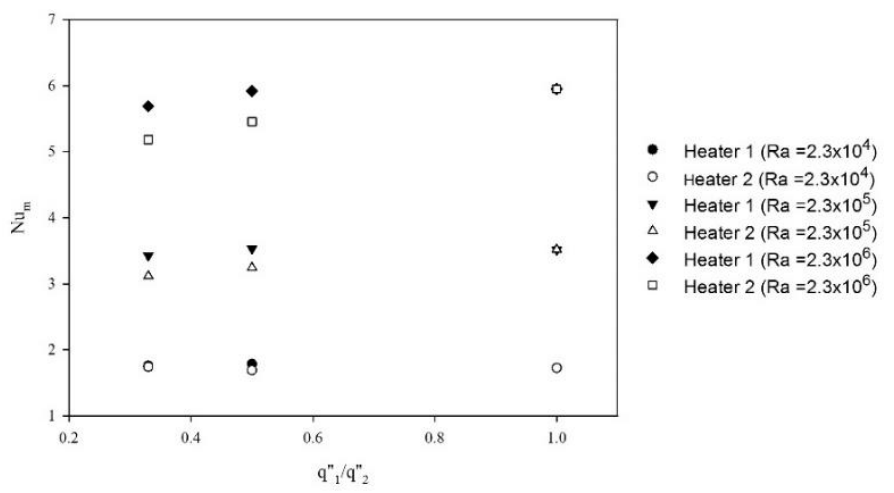

Fig. 8: Mean Nusselt number variation on the heater surfaces with heat dissipation ratio.

\section{Conclusion}

Two dimensional, laminar natural convection inside a rectangular cavity with two discrete heat sources placed on its bottom wall is investigated experimentally and numerically. Effects of the heat dissipation ratio and Rayleigh number on flow and heat transfer were investigated. The major findings of the present study can be presented as follows:

- Heat flow corridor and plume location changes with heat dissipation ratio and it is closer to the stronger heat source. 
- Mean Nusselt number values on both heater surfaces increase with Rayleigh number and decrease with heat dissipation ratio because of the decrease in recirculation strength.

- Maximum value of the mean Nusselt number is obtained on weaker heat source because of conduction heat transfer inside the enclosure walls.

- This study shows that conduction heat transfer cannot be neglected in the electronics cooling applications.

- Since, thermal conductivities of the plexiglass and FR-4 plates which is used as the PCB material are close to each other, plexiglass can be preferred as the test material for electronics cooling.

\section{Acknowledgements}

This study is supported by The Scientific and Technological Research Council of Turkey (TUBITAK) with project number of $114 \mathrm{M} 589$.

\section{References}

[1] O. Aydin, W. Yang, "Natural convection in enclosures with localized heating from below and symmetrical cooling from sides," International Journal of Numerical Methods for Heat \& Fluid Flow, vol. 10, no. 5, pp. 518-529, 2000.

[2] F. Corvaro, M. Paroncini, "Experimental analysis of natural convection in square cavities heated from below with 'D-PIV and holographic interferometry techniques," Experimental Thermal and Fluid Science, vol. 31, pp. 721-739, 2007.

[3] S. Banerjee, A. Mukhopadhyay, S. Sen, R. Ganguly. "Natural convection in a bi-heater configuration of passive electronic cooling," International Journal of Thermal Sciences, vol. 47, pp. 1516-1527, 2008.

[4] S. Saravanan, C. Sivaraj. "Natural convection in an enclosure with a localized non uniform heat source on the bottom wall," International Journal of Heat and Mass Transfer, vol. 54, no. 13-14, pp. 2820-2828, 2011.

[5] F. Zaman, T. Turja, M. Molla, "Buoyancy driven natural convection flow in an enclosure with two discrete heating from below," Procedia Engineering, vol. 56, pp. 104-111, 2013.

[6] S. G. Martyushev, M. Sheremet. "Conjugate natural convection combined with surface thermal radiation in an air filled cavity with internal heat source," International Journal of Thermal Sciences, vol. 76, pp. 51-67, 2014.

[7] G. Nardini, M. Paroncini, R. Vitali. "Experimental and numerical analysis of the effect of the position of a bottom wall hot source on natural convection," Applied Thermal Engineering, vol. 92, pp. 236-245, 2016.

[8] ANSYS Inc., ANSYS Fluent User's Guide Release 15., 2013.

[9] S. Kimura, A. Bejan, "The "Heatline" Visualization of Convective Heat Transfer," Journal of Heat Transfer vol. 105, no. 4, pp. 916-919, 1983.

[10] Q. Deng, G. Tang, "Numerical visualization of mass and heat transport for conjugate natural convection/heat conduction by streamline and heatline," International Journal of Heat and Mass Transfer, vol. 45, no. 11, pp. 2373$2385,2002$. 